\title{
Treatment of HCV in individuals with decompensated cirrhosis
}

Keywords: public health, gastroesophageal variceal hemorrhage, ascites, hepatocellular carcinoma, ns5b

Abbrevations: HCV, hepatitis c virus; SVR, sustained virological response; DAA, direct-acting antiviral agents; CTP, childturcotte-pugh

\section{Mini review}

Chronic hepatitis $\mathrm{C}$ virus (HCV) infection remains a public health challenge with an estimated global prevalence of $2.8 \%$, indicating that over 185 million people are infected worldwide, although this is likely an underestimate. The disease is generally slowly progressive and characterized by persistent hepatic inflammation that leads to cirrhosis in approximately $10-20 \%$ of individuals over $20-30$ years, with distinct inter individual variability in progression based on multiple variables. ${ }^{1}$ Once cirrhosis is established complications such as ascites, gastroesophageal variceal hemorrhage, hepatic encephalopathy, hepatocellular carcinoma, and/or acute or chronic liver failure may develop and result in diminished quality of life and survival without liver transplantation. Effective antiviral therapy that results in sustained virological response (SVR) is the only strategy that positively alters the natural history of liver disease associated with HCV infection by reducing the frequency of hepatic decompensation, liver-related mortality, all-cause mortality, need for liver transplantation, and hepatocellular carcinoma. ${ }^{2}$ Furthermore, SVR is also associated with improved quality of life and increased work productivity. ${ }^{3}$ Licensure of new generation direct-acting antiviral agents (DAAs) revolutionized treatment of HCV infection, as these agents have very high virological efficacy, low frequency of severe adverse events (AEs), and overall high barrier to resistance.

Hepatic decompensation is associated with markedly diminished survival and poor quality of life, thus liver transplantation remains the treatment of choice regardless of the etiology of liver disease. Nevertheless, the large imbalance between individuals in need for liver transplantation and supply of donor organs remains an important limitation. Antiviral therapy with interferon-based regimens was particularly challenging in this population as it resulted in severe toxicity and exceedingly poor SVR. In contrast, interferon-free regimens with DAAs have excellent tolerability and high virological efficacy in individuals with decompensated cirrhosis (Child-TurcottePugh [CTP] classes B and C), thus these agents have resurged interest of experienced practitioners in treating $\mathrm{HCV}$ infection in this population. Prior to initiating antiviral therapy with DAAs in individuals with decompensated cirrhosis it is important to assess and determine potential candidacy for liver transplantation. Conceivable benefits and risks of treating $\mathrm{HCV}$ infection pre- versus post-liver transplantation should be discussed in detail and some important clinical points unique to this population that must be addressed include:

a. Potential for improvement of biochemical parameters without significant improvement in quality of life following successful antiviral therapy (MELD limbo or purgatory)
Volume 3 Issue 4 - 2017

\author{
Andres F Carrion \\ Director of Hepatology, Texas Tech University Health Sciences \\ Center, USA
}

\begin{abstract}
Correspondence: Andres F Carrion MD, Director of Hepatology, Assistant Professor of Medicine, Texas Tech University Health Sciences Center, El Paso, Texas, 4800 Alberta Avenue, El Paso, Texas 79905, USA, Tel 9152155I42, Fax 9155456634,Email a.carrion@ttuhsc.edu
\end{abstract}

Received: March 23, 2017 | Published: August 10, 2017

\section{b. Use of HCV-infected grafts for liver transplantation}

c. Safety and efficacy of DAAs in decompensated cirrhosis versus post-liver transplantation.

Improvement of hepatic function is commonly observed during and after successful antiviral therapy with DAAs, even in individuals with severe hepatic decompensation (CTP class C), and is mainly reflected by reductions in serum bilirubin and prothrombin time resulting in lower MELD scores. ${ }^{4,5}$ All in all this is a positive outcome for individuals that also experience improved quality of life and diminished complications of cirrhosis; nevertheless, despite reductions in the MELD score following successful antiviral therapy for HCV some individuals may continue to experience poor quality of life and severe complications of cirrhosis. Organ allocation for liver transplantation in most countries is dictated by disease severity models such as the MELD score that incorporate biochemical parameters but do not account for impairment of quality of life, thus such reductions in biochemical parameters and consequently the MELD score following antiviral therapy for HCV may result in some individuals with poor quality of life due to liver-related complications to fall below the range of contention for liver transplantation. This clinical scenario has been termed "MELD limbo or MELD purgatory" and should be considered prior to starting antiviral therapy in individuals with decompensated cirrhosis and MELD scores approaching the range in which liver transplantation is a realistic option. ${ }^{6}$ The ongoing shortage of donor grafts along with robust data supporting the efficacy and safety of antiviral regimens with DAAs for treatment of recurrent HCV infection in liver transplant recipients has spearheaded a growing interest for offering $\mathrm{HCV}$-infected grafts to individuals with $\mathrm{HCV}$ infection listed for liver transplantation. Although no clinical trial to date has evaluated efficacy of antiviral regimens with DAAs following transplantation of HCV-positive liver grafts, outcomes are expected to be similar compared to treatment of recurrent $\mathrm{HCV}$ infection following transplantation of an $\mathrm{HCV}$-negative liver graft.

DAAs targeting various viral proteins such as the NS5B (sofosbuvir) and NS5A (ledipasvir, daclatasvir, velpatasvir) are in general well-tolerated by individuals with decompensated cirrhosis. ${ }^{4,5}$ In contrast, NS3/4A protease inhibitors such as simeprevir, grazoprevir, and paritaprevir are contraindicated in individuals with decompensated cirrhosis due to increased drug levels as well as post-marketing reports of worsening hepatic decompensation and 
liver failure in few individuals treated with agents in this class. If no contraindications exist (i.e., anemia), concomitant administration of ribavirin is recommended in all individuals with decompensated cirrhosis receiving antiviral therapy with regimens containing DAAs (regardless of the genotype) as it improves SVR. The recommended initial dose for ribavirin in individuals with severe hepatic decompensation (CTP class $\mathrm{C}$ ) is $600 \mathrm{mg}$ orally once daily, which can be subsequently increased as tolerated. If ribavirin is contraindicated, extending the duration of therapy from 12 to 24 weeks is an alternative for all regimens containing DAAs. Table 1 summarizes various antiviral regimens currently recommended by guidelines for use in individuals with decompensated cirrhosis. Antiviral therapy with sofosbuvir (NS5B nucleotide polymerase inhibitor) in combination with an NS5A inhibitor such as ledipasvir, daclatasvir, or velpatasvir along with concomitant ribavirin for 12 weeks results in SVR in 82$96 \%$ of individuals with HCV genotype 1 infection and decompensated cirrhosis. ${ }^{4,5,7}$

Table I Antiviral regimens with DAAs recommended for individuals with hepatic decompensation

\begin{tabular}{llll}
\hline \multirow{2}{*}{ DAAs } & HCV genotypes & \multicolumn{2}{l}{ Duration } \\
\cline { 3 - 4 } & DAAs plus ribavirin & DAAs without ribavirin \\
\hline Sofosbuvir/ledipasvir & 1,4 & 12 weeks & 24 weeks \\
Sofosbuvir/daclatasvir & I, 2,3 & 12 weeks & 24 weeks \\
Sofosbuvir/velpatasvir & I, 2, 3,4,5,6 & 12 weeks & 24 weeks \\
\hline
\end{tabular}

\section{DAAs, direct-acting antiviral agents}

In general, genotype 1a exhibits lower SVR compared to genotype 1b. CTP class also predicts response to antiviral therapy with CTP class B demonstrating higher SVR than CTP class C. Combination of sofosbuvir plus either daclatasvir or velpatasvir along with concomitant ribavirin for treatment of genotypes 2 and 3 results in SVR in $80-100 \%$ and $83-85 \%$, respectively. ${ }^{4,7}$ Although the number of individuals with genotype 4 and decompensated cirrhosis enrolled in clinical trials is very small, reported SVR following treatment with sofosbuvir plus ledipasvir, daclatasvir, or velpatasvir with concomitant ribavirin is $67-100 \%{ }^{4,5,7}$ Antiviral regimens containing sofosbuvir, an NS5A inhibitor (ledipasvir, daclatasvir, or velpatasvir), and ribavirin are associated with frequent AEs in individuals with decompensated cirrhosis (up to $100 \%$ may report AEs); nevertheless, these are overwhelmingly non-severe AEs such as fatigue, gastrointestinal intolerance, anemia, and headache. Severe AEs occur in $11-42 \%$ of individuals with decompensated cirrhosis treated with these regimens and discontinuation of DAAs due to AEs occurs in up to $8 \%$. Reported mortality during or shortly after completion of antiviral therapy with these agents is up to $3 \%$ for individuals with decompensated cirrhosis. Improvement of hepatic function as reflected by reductions in the CTP or MELD scores has been reported in up to $60 \%$ of individuals that achieve SVR and is more noticeable in those with higher baseline scores $(81 \%$ of patients with MELD score $\geq 15$ versus $51 \%$ of patients with MELD score $<15)$. $4,5,7$ Whether these improvements persist beyond short-term follow up remain to be documented; nevertheless, a recent population-based study showed a $32 \%$ decline in liver transplantation listings for HCV-related liver disease during 2014-2015 compared to previous years. These data suggest that reductions in MELD scores and overall complications of cirrhosis following antiviral therapy with DAAs are indeed sustained, at least for few years. In conclusion, availability of interferon-free regimens with DAAs permits treatment of $\mathrm{HCV}$ in individuals with decompensated cirrhosis with overall relatively high virological efficacy across different genotypes. Importantly, SVR rates are in general lower in individuals with decompensated cirrhosis compared to those seen in individuals with compensated cirrhosis (CTP class A) and liver transplant recipients (with or without cirrhosis but no hepatic decompensation). Furthermore, the frequency of AEs (including severe AEs) is markedly higher in this population, thus selection of candidates for antiviral therapy must be individualized in the setting of hepatic decompensation and treatment should only be administered by highly experienced practitioners, and ideally at a liver transplant center.

\section{Acknowledgements}

None.

\section{Conflict of interest}

Author declares that there is no conflict of interest.

\section{Consulting: Gilead Sciences}

Speaker: Merck, BMS

\section{References}

1. Westbrook RH, Dusheiko G. Natural history of hepatitis C. Journal of Hepatology. 2014;61(1):S58-S68.

2. Vander Meer AJ, Veldt BJ, Feld JJ, et al. Association between sustained virological response and all-cause mortality among patients with chronic hepatitis $\mathrm{C}$ and advanced hepatic fibrosis. JAMA. 2012;308(24):2584-2593.

3. Smith-Palmer J, Cerri K, Valentine W. Achieving sustained virologic response in hepatitis $\mathrm{C}$ : a systematic review of the clinical, economic and quality of life benefits. BMC Infect Dis. 2015;15:19.

4. Curry MP, O'Leary JG, Bzowej N, et al. Sofosbuvir and Velpatasvir for HCV in Patients with Decompensated Cirrhosis. $N$ Engl $J$ Med. 2015;373(27):2618-2628.

5. Charlton M, Everson GT, Flamm SL, et al. Ledipasvir and Sofosbuvir Plus Ribavirin for Treatment of HCV Infection in Patients With Advanced Liver Disease. Gastroenterology. 2015;149(3):649-659.

6. Carrion AF, Khaderi SA, Sussman NL. Model for end-stage liver disease limbo, model for end-stage liver disease purgatory, and the dilemma of treating hepatitis $\mathrm{C}$ in patients awaiting liver transplantation. Liver Transpl. 2016;22(3):279-280.

7. Poordad F, Schiff ER, Vierling JM, et al. Daclatasvir with sofosbuvir and ribavirin for hepatitis $\mathrm{C}$ virus infection with advanced cirrhosis or postliver transplantation recurrence. Hepatology. 2016;63(5):1493-1505. 